\title{
Instant Messaging Among Teens: An Exploratory Look at Gender and Time Usage
}

\author{
Jessica A. Lloyd, Peggy S. Meszaros, Ben Gotow ${ }^{1}$
}

\begin{abstract}
This study investigates gender differences, usage times, and peak times for adolescent instant messaging (IM) usage. Using a computer program called Buddy Tracker, data was collected on the actual IM usage of nearly 400 teens, ages 13-18. An Internet web-log (blog) community was also electronically observed to determine its gender composition. Gender differences were detected with girls outnumbering boys by nearly 2.6 to 1 on Xanga.com, However, among active IM users, males and females demonstrated almost identical usage times. (Males: 1 hour, 47 minutes: females: 1 hour 46 minutes.) Gender differences were noted, however, in IM usage habits. This work highlights the need for instant messaging research to employ naturalistic observation in addition to the previously relied upon data collection methods of self-reported interviews and focus groups.
\end{abstract}

Key Words: Gender, Instant Messaging, technology, teens, time usage

${ }^{1}$ Virginia Polytechnic Institute and State University. Electronic mail may be sent via Internet to jlloyd04@vt.edu.

It is a classic image deeply entrenched in the mind of the $20^{\text {th }}$ century American parent: an adolescent girl with a phone to her ear. Chatting with friends she left only moments ago, she paces the floor tethered to the wall by the 3-foot cord. In clear sight of her parents and siblings, her conversational content and time usage are subject to constant scrutiny and review. But in reality, the networking habits of today's teen bear little resemblance to this once classic image. The landline telephone has long been replaced by the cell phone (Pew Internet \& American Life Project, 2004) and the Internet, particularly instant messaging (IM), is well on its 
way to becoming the communication medium of choice for teens (Guidry, 2004; Pew Internet \& American Life Project, 2004; Grinter \& Palen, 2002).

Yet even as IM has become mired in the life of the American teenager (Guidry, 2004) many unanswered questions related to gender and time use remain. It has been determined, for example, that women are more relationship oriented than men (Boneva, et al., 2001), and that women invest more in personal relationships and maintain a more extensive social network (Moore, 1990; Walker, 1994; Wellman, 1992). Women are also more frequent users of the telephone (Walker, 1994) and heavier users of email (Boneva, Kraut \& Frohlich, 2001). But does it follow that adolescent girls are more likely to be heavy IM users than adolescent boys? Do girls participate in Internet web-blog communities, such as Xanga.com, more frequently than boys?

Grinter and Eldridge (2001) raised issues of public versus private space as they emphasized that the popularity of IM among teens stems from their need to socialize with friends while confined to their homes. Hellenga (2002) reinforces this notion, stating that peer-based connectedness is especially important for adolescents. Grinter and Palen (2002) further suggest that teens use IM to carve out a private world within the public space of home. Parents, by definition, are not included in this private space, thus it becomes difficult to determine just how much time their teens are spending on IM. Subrahmanyam, Kraut, Greenfield, and Gross (2000) have shown that most parents believe that purchasing a computer for their teen will give them an educational advantage, but if teens are multitasking between homework and IM, or remaining online IMing with friends long after their parents have gone to bed, this advantage will quickly disappear.

Using self-reported phone surveys and focus groups, the Pew Internet \& American Life Project (2005), an independent nonprofit organization, has provided a wealth of information about 
teens and the use of IM. As suggested by Reynolds (1971), however, this exploratory study utilized naturalistic observation via a computer program called Buddy Tracker. Written by a member of our research team, this innovative approach allowed us to monitor and record the IM usage of our sample population thereby providing a snap shot of actual, rather than self-reported, teen IM usage.

Numerous studies suggest that extended computer use among children may be linked to an increase risk of obesity, seizures, and hand injuries (Subrahmanyan, et al., 2000). Yet, the Pew Internet \& American Life Project (2005) highlights a growth in computer usage among teens of roughly $24 \%$ in the last five years. Clearly it is important to know how much time teens are actually spending online, particularly when utilizing IM.

\section{Literature Review}

Adolescence is defined by a strong need for numerous friendships and peer-group affiliations. (Boneva, Quinn, Kraut, Kiesler and Shlovski, in press.) In addition, this developmental period is typically defined by the need for person-to-person communication with friends (Boneva, et al., in press.). Kyratzi (2004) also emphasizes the need for peer communication as a means of establishing and maintain peer culture. She asserts that peer talk is an essential device teens utilize to display their identities and ideologies. Likewise, teens maintain a higher number of friends than adults (Berndt, Hawkins, \& Hoyle, 1986).

Instant messaging is one communication modality that adolescents use to satisfy this need (Boneva, et al., in press.). In fact, according to the Pew Internet \& American Life Project (2005), IM has become a staple of teens' daily lives, with threequarters of the teens online and $65 \%$ of all teens reporting that they use IM to communicate with their friends. Boneva, et al., (in press) found that IM satisfies two major needs for adolescents: 
maintaining individual friendships and belonging to peer groups. Instant messaging has become so popular that IM user names are more often exchanged by teens than phone numbers (Pew Internet \& American Life Project, 2005).

\section{Gender Differences in Internet Friendship Behaviors}

Subtahmanyam, et al., (2000) also found that teens especially girls- liked using the Internet for communicating with friends. In fact, women as a group spend significantly more time online than men (Pew Internet \& American Life Project, 2004), and many are now using the Internet to communicate with family and maintain social relationships with friends (Boneva, et al., 2001).

Research suggests that women tend to value relationships differently and to have different styles in sustaining them (Duck \& Wright, 1993: Spence \& Buckner, 1995; Aylor and Dainton, 2004). Women, for example, tend to spend time in intimate conversations with their good friends, whereas men tend to spend time in common activities with theirs (Duck \& Wright, 1993; Twenge, 1997; Walker, 1994).

The purpose of this study was to explore both gender differences and time spent by adolescents on IM usage. Past research has demonstrated that women are heavier users of email and the telephone, but does it follow that adolescent girls are more likely to be heavy IM users than adolescent boys? Likewise, do girls spend more time online and participate in Internet web-blog communities, such as Xanga.com, more frequently than boys? It was our hypothesis that they do.

\section{Method}

This exploratory study utilized naturalistic observation via a computer program to monitor the IM usage of male and female 
teenagers. Naturalistic observation was chosen as the methodology to insure against the exaggeration or minimization of data that may be associated with self-reported data collections. After conducting a 24-hour pilot study of 71 local high school students (30 girls and 41 boys) ages 13-18, four hundred teens (200 boys and 200 girls) were electronically observed while using IM for two 18-hour periods.

\section{Operational Definitions}

Instant Messaging (IM) is the term given to an Internetbased synchronous text chat with point-to-point communication between users on the same system (Grinter \& Palen, 2002). A typical user will participate in multiple written conversations with known friends on a pre-established buddy list (Subrahmanyam, et al. 2000).

Xanga.com is an Internet website that allows users to create a personal homepage and to enter blog-style discussion forums. The Xanga homepage provides 15 possible interest categories and allows users to connect with others who share their specific interest. During the sign up process, members are asked to enter personal information such as their birthdates, gender, and IM screen names. It was this information our crawler tracked.

A blogring is a group of web-logs (blogs) or user postings on a similar topic within an Internet community.

An Internet crawler is a computer program that independently follows links searching for hits or data points that match its predetermined selection criteria.

A heavy IM user, for the purpose of this study, shall be defined as a teen who records over four hours of IM usage during a collection period. 


\section{Participants}

A random sample of teenagers, ages 13-18, was obtained from the Internet website www.xanga.com. Once on the Xanga website, the category of People, sub-category of Friends was selected. On February $5^{\text {th }}, 2006$, the Friends category consisted of 310,337 possible blogrings. Data was collected by launching a specifically designed Internet crawler to gathered user information from three separate blogrings. During each collection interval, the crawler was instructed to search for and record an individual's birth date, gender, and IM screen name. Users who did not display this information were skipped over and not recorded as data. Likewise, users with birthdates outside the desired range (1987 1993) were also unrecorded. No personal information was collected.

The first blogring crawled was randomly selected from the Friends category and provided a sample of 1,100 girls and 290 boys. A second collection obtained from a randomly selected blogring, also in the Friends category, provided a collection of 1,235 girls to 515 boys. The third blogring, entitled Random High School Students, offered a sample of 332 girls and 220 boys. All participants were unaware of the presence of the crawler and no contact was made with any individual user.

These preliminary data sets were then sorted by birthdates and valid IM user names. Outliers and listings with insufficient data were discarded. Teens with mobile text messaging capabilities (as indicated by their "away" messages or constant active availability status) were also discarded. Due to the limitations of our Buddy Tracker program, this sample was further reduced to include a random sample of 200 males and 200 females. An additional sample set of 200 males and 200 females were retained for future research; all others were discarded. 


\section{Instruments}

Buddy Tracker is a publicly available Macintosh shareware program designed and created by a member of our research team. It allows the user to enter anywhere from 1 to 200 user names and tracks the IM usage of these individuals. Buddy Tracker classifies each individual's online availability status as active, idle, or away in a graphic fashion. It does not however, collect IP addresses or any personal information that has not been made publicly available by the adolescences themselves. In addition, the program does not attempt to monitor who is talking to whom, nor can it be used to make contact with an individual. Buddy Tracker is capable of recording and printing online activity data for extended periods, though for the purpose of this study, 18-hour intervals were utilized.

In addition to bar graphs, this program provides simple tables based on the number of active, idle, and away users over time. It also provides detailed numerical information about how long an individual spent in each of the three categories. (See Figure 1.)

\section{Data Collection}

Boys

Buddy Tracker data was collected on a final sample of 200 boys ages $13-18$. The first data collection was run from 3 p.m. on Monday, February 6, 2006 until 7a.m.on Tuesday, February 7, 2006. The second collection took place from 2 p.m. on Tuesday, February 7, 2006 until 7 a.m. on Wednesday, February 8, 2006. After collecting the data, six boys, who were recorded as having mobile text messaging and/or displayed more than seven hours of active IM use, were removed from our sample. 
Girls

Buddy Tracker data was collected on a final sample of 200 girls ages $13-18$. The first data collection was run from 3 p.m. on Tuesday, February 14, 2006 until 7a.m. on Wednesday, February 15, 2006. The second collection took place from 2 p.m. on Wednesday, February 15, 2006 until 7a.m. on Thursday, February 16, 2006. After collecting the data, 12 girls, who were recorded as having mobile text messaging and/or displayed more than 7 hours of active IM use, were removed from our sample.

\section{Results}

In keeping with past gender trends, more girls than boys were members of Xanga.com. In fact, after launching the crawler on the Xanga website, girls were found to outnumber boys in one sampling by nearly 4 to 1 (1100 girls to 290 boys). This finding was upheld in two additional blogrings, one selected again at random from the friends category ( 1235 girls to 515 boys: a ratio of nearly 2.5 to 1 ), and one selected from a ring entitled: Random High School Students, (332 girls and 220 boys: a ratio of 1.5 to 1). This finding confirms the work of Boneva, et al., (2001), Turow, (1999) and Subrahmanyam and Greenfield, (1998).

In our largest sampling of 3,286 mixed gender users of Xanga.com, it was determined that approximately $53 \%$ of those analyzed had also provided their personal IM user names $(\mathrm{n}=$ 1,750). It must be noted however, that some IM names were clearly fictitious. Invalid word strings such as "Imnottelling" (I'm not telling), for example, were occasionally recorded by our crawler as correct. This limitation will skew our data slightly, and thus these results can be viewed only as approximations.

Additionally, because participants in chat rooms (or blogrings) are anonymous and can provide misleading information about themselves, it is impossible to provide a reliable 
ethnographic description of the participants (Schönfeldt \& Golato, 2003). It must also be noted that while more than half (52\%) of all teens have only one screen name, $22 \%$ of teens report that they have two (Pew Internet \& American Life Project, 2005). Old screen names are often remnants of earlier years which the teen has not bothered to delete. Though our Internet crawler was able to detect and ignore similar names or variations in capitalization, hyphens or spaces among the same name, some outdated or inactive names were inevitably picked up by our crawler and recorded as valid.

It was also noted that, on average, each user listed roughly 25 additional friends. This finding is in line with the Pew Internet \& American Life Project (2005), which reported that the majority $(36 \%)$ of teens had less than 25 friends on their buddy list. Due to the limitations of our crawler, however, this information could not be collected on a gender basis. Nor was it possible to infer how many friend links were accurate or associated with each individual blog.

\section{Boys: Active and Inactive Users}

Buddy Tracker data was collected on a final sample of 194 boys. This data revealed that the average amount of time our total sample $(n=194)$ spent online was one hour. For active users only $(n=110)$, however, this number rose to 1 hour and 47 minutes. The shortest recorded IM usage was three minutes. The longest IM availability time recorded was 6 hours and 26 minutes. The most popular time for male teens to be online was 9:30 p.m. with 41 users active in both data sets. The largest user drop-off occurred between 10:30 p.m. and 11:00 p.m. when the number of active users dropped from 33 to 17 respectively. An average of 11 users remained online after midnight.

When looking at both active and inactive male users $(\mathrm{n}=$ 194), the largest percentage $(21 \%)$ used IM for more than two 
minutes but less than an hour during our collection interval. Twelve percent were online for more than an hour and less than two hours. Eight percent were online for more than two hours but less than three, and 5\% were on for more than three hours but less than four. Four percent of the male teens sampled remained active on IM for over 5 hours. (See Table 1.)

\section{Boys: Active Users Only}

Of our active male users $(n=110)$, again the largest percentage of male teens $(37 \%)$ were online for more than two minutes but less than an hour, while $22 \%$ were online for more than an hour but less than two. Seven percent of active male IM users were recorded as active for more than five hours (See Table 2).

\section{Girls: Active and Inactive Users}

Buddy Tracker data was then collected on a final sample of 184 girls. This data revealed that the average amount of time our total female sample $(n=184)$ spent online was 48 minutes. For active users only $(n=86)$, however, this number rose to 1 hour and 46 minutes. The shortest recorded IM usage was one minute. The longest IM availability time recorded was 6 hours and 34 minutes. The most popular time for girls to be online was 7:30 p.m. No dramatic user drop-off occurred for girls. Instead user numbers slowly declined after 7:30 p.m. An average of 10 users remained online after midnight.

When looking at both active and inactive female users, $(n=$ 184), Fourteen percent used IM for more than two minutes but less than an hour during our collection interval. Nine percent were online for more than an hour and less than two hours. While 5\% were online for more than two hours but less than three, and $2 \%$ were on for more than more than three hours but less than four. 
Two percent of female teens remained active on IM for over 5 hours (see Table 1).

Table 1. Percent of Active and Inactive IM Users Online

\begin{tabular}{|lcc|}
\hline Using IM for: & Male Users: & Female Users: \\
More than 2 but less than 60 min. & $21 \%$ & $14 \%$ \\
More than 1 but less than 2 hours & $12 \%$ & $9 \%$ \\
More than 2 but less than 3 hours & $8 \%$ & $5 \%$ \\
More than 3 but less than 4 hours & $5 \%$ & $2 \%$ \\
More than 4 but less than 5 hours & $4 \%$ & $1 \%$ \\
More than 5 hours & $4 \%$ & $2 \%$ \\
\hline
\end{tabular}

\section{Girls: Active Users Only}

Of our active female users $(n=86)$, the largest percentage $(40 \%)$ were online for more than two minutes but less than an hour, while $25 \%$ were online for more than an hour but less than two. Six percent of active female IM users were recorded as active for more than five hours. (See Table 2.)

\section{Discussion}

The information collected by our Internet crawler concurs with our expectations and the current literature. Girls were in fact more frequent users of the Internet web-blog community Xanga.com, outnumbering their male counterparts by an average ratio of nearly three (2.6) to one. This finding is well supported by the literature (Boneva, et al., 2001; Duck \& Wright, 1993; Moore, 1990; Spence \& Buckner, 1995; Walker, 1994). 
The information collected by our Buddy Tracker program, however, did not find girls to be generally more active IM users than boys. In fact, among teens who actively used IM, males and females were found to demonstrate almost identical usage times. The average active male spent 1 hour and 47 minutes online, while the average active female spent 1 hour 46 minutes online. A noticeable difference was displayed, however, when considering the total sample population. Among all teens sampled, boys on average, spent one hour a day actively using IM, while girls averaged only 48 minutes per day. This finding was also noted in our pilot study. Another difference occurred in the number of heavy IM users. In our female population, nine users exceeded five hours of IM time over the two collection intervals; among boys however this number nearly doubled as 16 users fell within this range. In fact, as IM usage times rose beyond two hours, the percentages of boys recorded in each category consistently surpassed girls. Fifteen percent of active males, for example, demonstrated over four hours of use, while only $11 \%$ of active females did the same.

\section{Table 2. Percent of Active IM Users Online}

\begin{tabular}{|lcc|}
\hline Using IM for: & Male Users: & Female Users: \\
More than 2 but less than 60 min. & $37 \%$ & $40 \%$ \\
More than 1 but less than 2 hours & $22 \%$ & $25 \%$ \\
More than 2 but less than 3 hours & $15 \%$ & $14 \%$ \\
More than 3 but less than 4 hours & $9 \%$ & $8 \%$ \\
More than 4 but less than 5 hours & $7 \%$ & $4 \%$ \\
More than 5 hours & $7 \%$ & $6 \%$ \\
\hline
\end{tabular}


Little research has been done on gender differences and adolescent IM usage. It has been found, however, that girls prefer email, (Pew Internet and American Life Project, 2005) and that girls have traditionally favored more open and intimate conversations (Boneva, et al., 2001; Aylor \& Dainton, 2004). Thus, it is quite possible that the short cryptic style of instant messaging fits the natural male preference for concise communication (Aylor \& Dainton, 2004: Baron, 2005).

In addition, the Pew study demonstrates that $51 \%$ of online teens continue to prefer the telephone when communicating with friends. As women have long been known to be heavy phone users (Walker, 1994), perhaps adolescent girls, as a whole, are supplementing their IM usage with phone time or face-to-faceinteractions.

More unsettling perhaps, is the number of teens, particularly boys, who were recorded as active on IM for extended periods of time (over four hours). Two factors may have influenced this finding. The first is the potential presence of an unanticipated technology which may have inadvertently resulted in Buddy Tracker recording inaccurate information. Although teens with mobile text messaging capabilities were removed from our sample, it is possible that other technologies have interfered with this program's ability to distinguish between active and idle usage.

The second factor which may have influenced our data stems from our initial sample being collected from Xanga.com, an Internet web-blog community. It is possible that using Xanga provided a population of highly connected teens with larger than average IM buddy lists. To test for this potential bias we referred to our pilot study where 71 students at a local high school were tracked with Buddy Tracker for a 24-hour period. This control sample also recorded several users, both male and female, logging over four hours of active IM time. However, no participant exceeded five hours of use. Thus, we conclude that IM usage 
times in the four-hour range may be accurate, while those exceeding five and even six hours remain subject to question.

Clearly more research is needed to resolve this ambiguity. However, we believe that teens, like adults, fall into one of two categories: heavy IM users and light IM users. Some teens, particularly boys, may be "hooked" on IM, spending up to, if not over, four hours a day IMing with friends.

Finally, it must be acknowledged that these findings consistently indicate more active IM usage by both males and females than was reported by the Pew Internet \& American Life Project (2005). We believe this discrepancy to be the result of our differing data collection methods. While the Pew study utilized self-reported data, we were able to naturalistically observe and record teens actual using IM. Teens may have inadvertently or intentionally underestimated their IM usage during the Pew interviews or reported the actual time they spent conversing (typing directly) with friends, while Buddy Tracker recorded the total time teens remained online with an active status.

Computer based communication may allow teens to escape the tether of the landline phone, but computers have in no way decreased the call for parental monitoring. There is a need for future research on gender and adolescent IM usage, particularly in terms of the number of teens, predominantly males, who appear to be using IM for longer periods of time. Such research, however, should aim to employ naturalistic observations as well as teen interviews or focus groups to insure an accurate data collection. Further research is also needed to understand the implications of teen time spent instant messaging versus face-to-face communication and development of personal relationships.

But, for now, please excuse us - we have an Instant Message waiting. 


\section{References}

Aylor, B., \& Dainton, M. (2004). Biological sex and psychological gender as predictors of routine and strategic relationship maintenance. Sex Roles: A Journal of Research, 50, 9-10 689699.

Baron, N. (2005, February). Instant messaging by American college students: A case study in computer-mediated communication. Paper presented at the American Association for the Advancement of Science Annual Meeting, Washington, DC.

Berndt, T. J., Hawkins, J. A., \& Hoyle, S. G. (1986). Changes in friendship during a school year: Effects on children and adolescents' impressions of friendship and sharing with friends. Child Development, 57, 1284-1297.

Boneva, B., Kraut, R., \& Frohlich, D. (Nov 2001). Using e-mail for personal relationships: the difference gender makes. (Community Studies of the Internet). In American Behavioral Scientist, 45, 530(1). Retrieved January 29, 2006, from Expanded Academic ASAP via Thomson Gale: http://find.galegroup.com/ezproxy.lib.vt.edu:8080/itx/infomark .do?\&contentSet=IAC-

Boneva, B., Quinn, A., Kraut, R. Kiesler, S., Cummings, J., Shklovski, I. (In press). Teenage communication in the instant messaging era. In R. Kraut, M. Brynin, and S. Kiesler (Eds). PCs, Phones, and the Internet: The Social Impact of Information.

Duck, S., \& Wright, P. H. (1993). Reexamining gender differences in friendships: A close look at two kinds of data. Sex Roles, 28, 709-727. 
Grinter, R., \& Palen, L. (2002). Instant Messaging in teen life. Proceedings of the 2002 ACM Conference on Computer Supported Cooperative Work. Retrieved October 15, 2004, from

http://portal.acm.org/ft_gateway.cfm?id=587082\&type=pdf\&c oll=Portal $\& \mathrm{dl}=\mathrm{ACM} \& \mathrm{CFID}=29733676 \& \mathrm{CFTOKEN}=5142097$ 3.

Grinter, R., \& Eldridge, M. (2001). Y do tngrs luv 2 txt msg?. Proceedings of ECSCW'01, Bonn, Germany, 219-238.

Guidry, K. (2004, November). Instant messaging: Its impact on and recommendations for student affairs. Student Affairs Online, 5, (4).

Hellenga, K. (2002). Social space, the final frontier: Adolescents on the Internet. In J. Mortimer \& R. W. Larson (Eds.). The changing adolescent experience: Societal trends and the transition to adulthood (pp. 208-249). New York: Cambridge University Press.

Kyratzi, A. (2004). Talk and interaction among children and the co-construction of peer groups and peer culture. Annual Review of Anthropology, 33, 625-649.

Moore, G. (1990). Structural determinants of men and women's personal networks. American Sociological Review, 55, 726735 .

Pew Internet and American Life Project. (2004, September 1).

How Americans use Instant Messaging. Retrieved October 15, 2004, from http://www.pewinternet.org/pdfs/PIP_Instantmessage_Report.p df. 
Pew Internet and American Life Project. (2005). Teens and Technology. Retrieved February 1, 2006, from http://www.pewinternet.org/pdfs/PIP_Teens_Tech_July2005w eb.pdf.

Reynolds, P. D. (1971). A primer in theory constructions. New York: Bobles-Merril.

Schönfeldt, J. \& Golato, A. (2003). Repair in chats: A conversation analytic approach. Research on Language and Social Interaction, 36(3), 241-284.

Spence, J. T., \& Buckner, C. (1995). Masculinity and femininity: Defining the undefinable. In P. J. Kalbfleish \& M. J. Cody (Eds.), Gender, power, and communication in human relationships (pp. 105-140). Hillsdale: NJ: Lawrence Erlbaum.

Subrahmanyam, K., \& Greenfield, P. M. (1998). Computer games for girls: What makes them play? In J. Cassell \& H. Jenkins (Eds.) From Barbie to Mortal Kombat: Gender and computer games. Cambridge, MA: MIT Press.

Subrahmanyam, K., Kraut, R.E., Greenfield, P.M., \& Gross, E.F. (2000, Fall/Winter). The impact of home computer use on children's activities and development. Children and Computer Technology, 10 (2), 123-144.

Turow, J. (1999, May). The Internet and the family: The view from the parents, the view from the press. Philadelphia: Annenberg Public Policy Center, University of Pennsylvania.

Twenge, J. M. (1997). Changes in masculine and feminine traits across time: A meta-analysis. Sex Roles, 36, 305-327. 
Walker, K. (1994). "I'm not friends the way she's friends": Ideological and behavioral constructions of masculinity in men's friendships. Masculinities, 2, 38-55.

Wellman, B. (1992). Men in networks: Private communities, domestic friendships. In P. M. Nardi (Ed.), Men's friendships (pp. 74-114). London: Sage. 